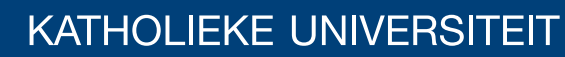 \\ LEUVEN
}

\section{Faculty of Business and Economics}

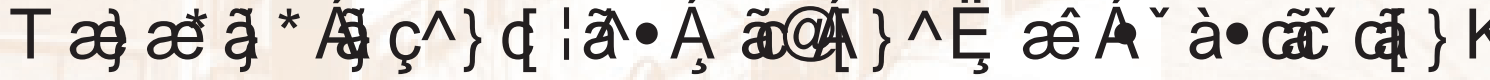
DIQHZ VMHQQRUDQDOVV

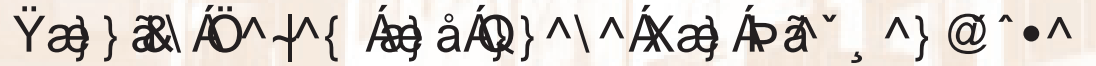

DEPARTMENT OF DECISION SCIENCES AND INFORMATION MANAGEMENT (KBI) 


\title{
Managing inventories with one-way substitution: a newsvendor
}

\section{analysis}

\author{
Yannick Deflem*, Inneke Van Nieuwenhuyse \\ Research Center for Operations Management, Department of Decision Sciences and Information \\ Management, K.U.Leuven, Belgium
}

\begin{abstract}
This paper presents a newsvendor approach to determine optimal order-up-to levels in a two-item inventory system with one-way substitution, assuming that both items are managed according to a periodic base stock order policy. The objective is to minimize the expected total cost per period, which consists of expected purchasing costs, expected inventory holding costs, expected shortage costs and expected adjustment costs. It is shown that, for any arbitrary (bivariate) continuous demand distribution, the optimal solution is unique. Moreover, the model yields useful insights on the impact of substitution on service level, the optimality of a borderline case in which the order-up-to level of the inflexible item is reduced to zero, and the pivotal role of the purchasing cost.
\end{abstract}

Keywords: inventory management, one-way substitution, newsvendor

\section{Introduction}

In many supply chains, mismatches between supply and demand are (at least partially) mitigated by keeping inventories, possibly at different levels of the supply chain (e.g., raw materials, components, semi-finished products and/or end items). The task of inventory management is to balance the benefits of inventory (i.e., reducing lost sales) with the associated cost (which is typically reflected in the inventory holding cost).

\footnotetext{
${ }^{*}$ Corresponding author: Yannick Deflem, Naamsestraat 69, 3000 Leuven, Belgium; Tel.: +32 16326577
}

E-mail addresses: yannick.deflem@econ.kuleuven.be and inneke.vannieuwenhuyse@econ.kuleuven.be 
One way of reducing the cost associated with inventory is to pool the demands of multiple items on the same (flexible) inventory item: provided that demands are not perfectly positively correlated, this allows a reduction in the required amount of safety stock, and (hence) a reduction in inventory holding cost. This is referred to as "risk-pooling" or "statistical economies of scale" [13]. However, flexibility tends to come at a cost: this "flexibility cost" can boil down to a product cost premium (when the flexible item is inherently more expensive to manufacture or purchase) and/or an additional adjustment cost (when the item needs to undergo additional processing or transportation in order to make it "fit for use" when demand arises).

This observation has spurred research on so-called substitution (or "tailored pooling") systems, in which flexible (and hence, more expensive) stock is used as a substitute only when the regular (cheaper) item stocks out. Tailored pooling can be obtained in a variety of ways, a.o. through the use of manufacturerdriven one-way substitution (e.g. [1], [10]), lateral transshipments (e.g. [9], [6]) and tailored postponement [11]. It offers a compromise between a setting with "full pooling" (implying that demand for a particular product type is always rerouted to the stock of the flexible product, and no productspecific stock is held) and "no pooling" (only product-specific stock is held, and demand can never be rerouted to stock of a different item).

In general, determining the optimal inventory control parameters in systems with substitution is complex: demands are only "partially pooled" on the inventory of the flexible item, and the amount of demand that can be "rerouted" to the flexible item depends on the order policies of both the dedicated product and the substitute. The optimal inventory control parameters are influenced by many different factors, such as the replenishment lead time (deterministic -- zero or strictly positive -- or stochastic), and the demand structure (demand distributions and correlation between the demands).

In this paper, we use a newsvendor approach to analyze the optimal order-up-to levels in a two-item inventory system with one-way substitution, assuming that both items are managed according to a 
periodic base stock order policy ${ }^{1}$. Only one item can be used as a substitute for the other (hence the term one-way substitution). The objective is to minimize the expected total cost per period, which consists of expected purchasing costs, expected inventory holding costs, expected shortage costs and expected adjustment costs. We first consider a decision maker who aims to optimize the order-up-to levels for both items in a single-period setting; next, we extend the model to a multiperiod setting.

Though our approach builds heavily on the work by Van Mieghem (see e.g. [12]), it differs in the sense that we consider the substitute (i.e., flexible item) to be an item in its own right, with its own demand to fulfill. Moreover, whereas the work by Van Mieghem studies flexible capacity, we apply the approach to an inventory setting, which yields useful insights a.o. on the effect of one-way substitution on customer service levels, and on the optimality conditions for the borderline case in which the order-up-to level of the inflexible product is reduced to zero. Throughout the analysis, we assume zero replenishment lead times (as common in the literature, see e.g. [8], [1] and [3]).

In Section 2, the research problem is described in further detail. The single-period newsvendor model is explained in Section 3. Section 4 extends the newsvendor to a multiperiod setting. Finally, Section 5 summarizes the main conclusions.

\section{Problem description}

Consider a setting with two different product types (Product 1 and Product 2) as in Figure 1.

Demand $d_{i, t}$ for a specific product type $i$ in period $t$ is preferably satisfied by means of its corresponding (product-specific or dedicated) inventory. The amount of demand that is fulfilled by dedicated inventory in period $t$ is represented by $x_{i, t}$ and indicated by the solid arrows in Figure 1.

\footnotetext{
${ }^{1}$ Since we do not optimize the review period (commonly referred to by $T$ or $R$ in the literature) we prefer to denote our policy as a base stock policy instead of using the (more common) $(R, S)$ notation.
} 


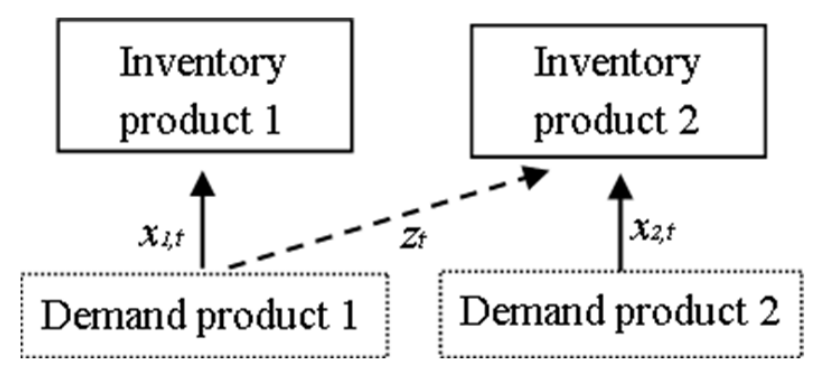

Fig. 1: A two-product inventory system with one-way substitution

Only when the dedicated inventory for product 1 is out of stock, demand can be satisfied by the substitute item (item 2). As such, part of the demand for item 1 can be "rerouted" to stock of item 2. This rerouted demand is indicated by the notation $z_{t}$ (see the dashed arrow in Figure 1); each unit of rerouted demand incurs a unit adjustment cost $a$.

Both inventories are managed according to a periodic base stock inventory policy. In a single-period setting, it is common to assume that the starting inventory position is zero (e.g., [7], [4], [8] and [12]) whereas in a multiperiod setting, the inventory position at the start of a period $t$ is equal to the inventory position at the end of period $t-1$. At the start of every period $t$, the decision maker places an order such that the inventory position is raised to the order-up-to level $S_{i}$ (for $i=1,2$ ) [2]. Since the replenishment leadtime is assumed to be zero, orders are received immediately; consequently, the net inventory immediately rises to $S_{i}$ once an order for item $i$ has been placed. The unit purchasing cost is represented by $c_{i}$ for $i=1,2$. At the end of every period t, the decision maker optimally allocates the observed demand to the different inventories, constrained by his earlier inventory investments (i.e., determine $x_{1, t}, x_{2, t}$ and $z_{t}$ ). Any leftover inventory of product $i$ at the end of the period incurs a unit holding $\cos t h_{\mathrm{i}}$. Demand of product $i$ that cannot be satisfied at the end of a period is penalized at a unit shortage cost $p_{\mathrm{i}}$ and is backordered to the next period (in the multiperiod model) or lost (in the single-period model). 


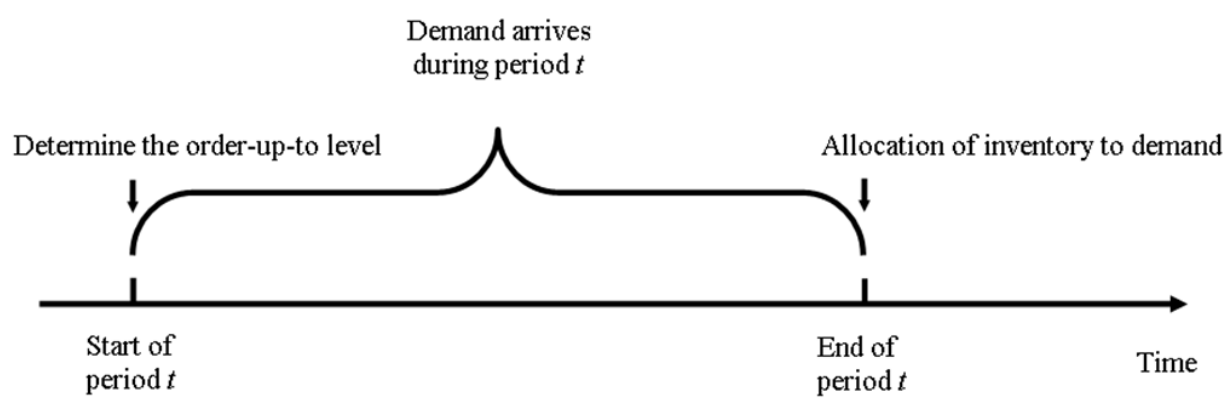

Fig. 2: Sequence of activities in an arbitrary period

As shown in Figure 2, this type of problem is a two-stage stochastic program problem. In a first stage, before demand is known, the optimal order-up-to levels are determined. In a second stage, once demand has been observed, the optimal allocation decision is made. The aim is to determine the optimal order decisions such as to minimize the total expected cost (i.e., sum of expected purchasing costs, expected shortage costs, expected inventory holding costs and expected adjustment costs).

A summary of the cost parameters, random variables and decision parameters is given in Table 1 .

\begin{tabular}{|l|l|}
\hline \multicolumn{2}{|l|}{ Cost parameters } \\
\hline$c_{\mathrm{i}}$ & Purchasing cost per unit of product $i$ \\
\hline$p_{\mathrm{i}}$ & Shortage cost per unit of unsatisfied demand of product $i$ at the end of the period \\
\hline$h_{\mathrm{i}}$ & Holding cost per unit of product $i$ left over at the end of the period \\
\hline$a$ & Adjustment cost per unit of demand for product 1 satisfied by product 2 \\
\hline Random variables \\
\hline$d_{i, t}$ & Demand during period $t$ of product $i$ \\
\hline$x_{i, t}$ & Amount of inventory of product $i$ allocated to demand of product $i$ in period $t$ \\
\hline$z_{t}$ & Amount of inventory of product 2 allocated to demand of product 1 in period $t$ \\
\hline Decision parameters \\
\hline$S_{1}$ & Order-up-to level of product 1 \\
\hline$S_{2}$ & Order-up-to level of product 2 \\
\hline
\end{tabular}

Table 1: Notation 
The cost parameters and demand distributions are assumed to remain constant over time, implying that the optimal order-up-to levels will also remain constant over time. Throughout this paper, the notation $\mathrm{E}[X]$ refers to the expected value of $X$ and $E[X]^{+}=E[\max (0, X)]$.

For a meaningful analysis, the following assumptions need to hold:

Assumption 1: $c_{2}-c_{1}+a>0$

The flexibility cost, which can be decomposed in a product cost premium and an adjustment cost, is strictly positive. This ensures that demand of product 1 is preferably satisfied with its corresponding inventory.

Assumption 2: $p_{1}+h_{2}>a+c_{2}-c_{1}$

In case of a shortage of product 1 and leftover inventory of product 2, it is more expensive to do nothing and incur $p_{1}+h_{2}$ than to use product 2 as a substitute and incur the associated flexibility cost. This condition ensures that it is optimal to reroute unsatisfied demand of product 1 to remaining stock of product 2 (if any).

Assumption 3: $h_{1}+a>h_{2}$

Transforming leftover inventory of product 2 into inventory of product 1 is never cost beneficial.

Assumption 4: $p_{2}+a>p_{1}$

The inventory of product 2 is preferably used to cover demand for product 2 .

Assumption 5 (only required for the single-period newsvendor model): $p_{i}>c_{i}$ 
Purchasing product $i$ is less expensive than incurring a penalty cost for that product. This assumption is needed in a single-period setting to avoid a solution in which it is preferable not to meet any demand ${ }^{2}$.

\section{Single-period newsvendor model}

Section 3.1 derives the newsvendor condition for the single-period system with one-way substitution. In Section 3.2, we derive a similar condition for the setting with $S_{1}=0$. The results imply that a threshold purchasing cost exists for product 1: as long as the purchasing cost of product 1 stays below this threshold, one-way substitution is optimal. Note, that because of assumption 2, a setting that does not allow any demand of product 1 to be rerouted can never be optimal.

\subsection{One-way substitution}

As mentioned in Section 2, our objective is to determine the values of $S_{1}$ and $S_{2}$ in order to minimize the expected total cost during a single period (denoted by $E[T C]$ ). This expected total cost can be calculated as $^{3}:$

$E[T C]=c_{1}\left(S_{1}\right)+c_{2}\left(S_{2}\right)+h_{1} E\left[S_{1}-d_{1}\right]^{+}+h_{2} E\left[S_{2}-d_{2}-\mathrm{z}\right]^{+}+p_{1} E\left[d_{1}-S_{1}-\mathrm{z}\right]^{+}+$

$p_{2} E\left[d_{2}-S_{2}\right]^{+}+a E[\mathrm{z}]$

Where $\mathrm{z}=\min \left\{\max \left(0, S_{2}-d_{2}\right), \max \left(0, d_{1}-S_{1}\right)\right\}$

Expression (1) can be reformulated in terms of the allocation variables $x_{1}, x_{2}$ and $z$ :

$E[T C]=c_{1}\left(S_{1}\right)+c_{2}\left(S_{2}\right)+h_{1} E\left[S_{1}-x_{1}\right]+h_{2} E\left[S_{2}-x_{2}-z\right]+p_{1} E\left[d_{1}-x_{1}-z\right]+p_{2} E\left[d_{2}-x_{2}\right]+$ $a E[z]$

\footnotetext{
${ }^{2}$ Note that this assumption is not needed in a multiperiod setting with backordering, since the amount of demand backordered is included in the replenishment order quantity, and fulfilled in the next period (if $S_{i} \geq 0$ ).

${ }^{3}$ As this section only considers a single period, subscript $t$ is omitted.
} 
The first two terms in expression (1) and (2) refer to the expected purchasing costs: as we assume that the starting inventory is zero, these are fully determined by the choice of $S_{1}$ and $S_{2}$. The third and fourth term represent the expected holding costs of leftover inventory at the end of the period: note that the allocation variables $\left(x_{1}, x_{2}\right.$ and $\left.z\right)$ vary with demand, and hence are random variables. The next two terms represent the expected penalty costs for lost sales, while the last term refers to the expected flexibility cost incurred by rerouting demand.

Recall that the problem is a two-stage decision process. In the first stage, the order-up-to levels $\left(S_{1}\right.$ and $\left.S_{2}\right)$ are determined and in the second stage, inventory is allocated to the demands $\left(x_{1}, x_{2}\right.$ and $\left.z\right)$.

In fact, for given order-up-to levels and given demands, the optimal allocations in the second stage $\left(x_{1}^{*}\right.$, $x_{2}^{*}$ and $z^{*}$ ) coincide with the solution to the following linear programming model (LP1)

$\operatorname{Min} c_{1}\left(S_{1}\right)+c_{2}\left(S_{2}\right)+h_{1}\left(S_{1}-x_{1}\right)+h_{2}\left(S_{2}-x_{2}-z\right)+p_{1}\left(d_{1}-x_{1}-z\right)+p_{2}\left(d_{2}-x_{2}\right)+a z$

s.t.

$$
\begin{gathered}
x_{1} \leq S_{1} \\
x_{2}+z \leq S_{2} \\
x_{1}+z \leq d_{1} \\
x_{2} \leq d_{2} \\
x_{1}, x_{2} \text { and } z \geq 0
\end{gathered}
$$

Given the assumptions imposed on the cost parameters (see Section 2), the optimal allocation resulting from (LP1) coincides with the allocation structure discussed in Section 2: allocate as much as possible of the demand to the dedicated stock, and reroute (if possible) the remaining demand for product 1 to remaining stock of product 2 (rather than lose the sale). The optimal objective function of (LP1) then reflects the total cost for given demand observations and order-up-to levels and is denoted by

\footnotetext{
${ }^{4}$ A necessary condition for which a feasible solution exists is $S_{1} \geq 0$ and $S_{2} \geq 0$.
} 
$T C\left(S_{1}, S_{2}, d_{1}, d_{2}\right)$. According to linear programming theory, $T C\left(S_{1}, S_{2}, d_{1}, d_{2}\right)$ is convex in $S_{1}$ and $S_{2}$ for a given $d_{1}$ and $d_{2}$ (see Appendix A for a formal proof).

Since the expected total cost $E[T C]$ is merely a weighted linear combination of $T C\left(S_{1}, S_{2}, d_{1}, d_{2}\right)$ over all possible demand realizations with the demand probabilities as weights, it follows that $E[T C]$ is also convex in $S_{1}$ and $S_{2}$. Therefore, the optimal $S_{i}^{*}$ are unique and can be found as the solution to the first order conditions $\partial E[T C] / \partial S_{i}=0(i=1,2)$.

Determining the first-order derivative of $E[T C]$ to $S_{1}$ and $S_{2}$, is analogous to calculating the expected shadow price of the first two constraints of (LP1) (see [5]): $\partial E[T C] / \partial S_{i}=E\left[\lambda_{i}\right](i=1,2)$. Consequently, the optimal $S_{1}^{*}$ and $S_{2}^{*}$ can be found by determining the expected shadow prices of these constraints, and requiring $E\left[\lambda_{1}\right]=E\left[\lambda_{2}\right]=0$.

The power of this result lies in its simplicity, as well as in its graphical interpretation: for any given combination of order-up-to levels $S_{1}$ and $S_{2}$, the demand space can be divided into 5 domains $\Omega_{j}$ (with $j=$ 0 to 4 ) with constant shadow prices $\lambda_{i j}$ (i.e. the shadow price of constraint $i$ for demand in domain $j$ ) as shown in Figure 3. Consequently, it is sufficient to calculate the shadow price for these 5 domains in order to find the expected shadow price.

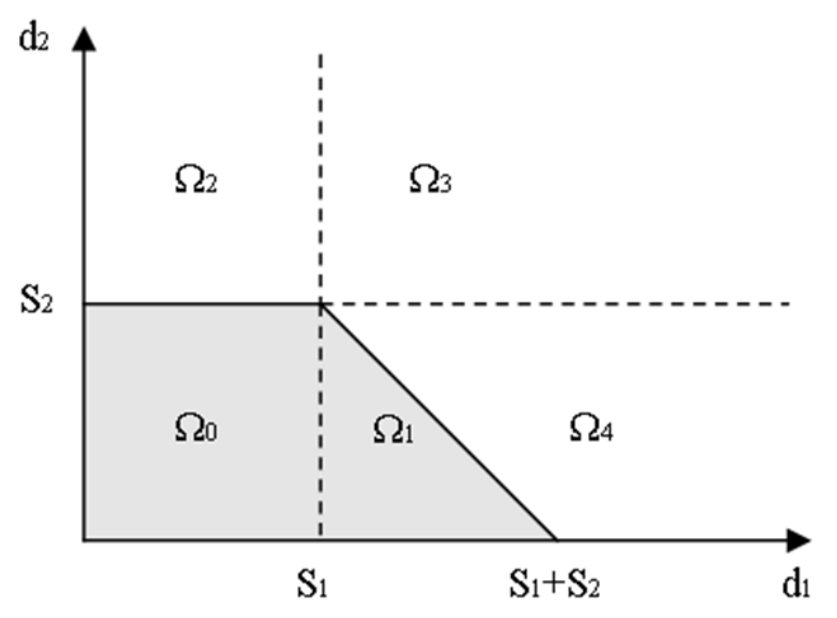

Fig. 3: Demand domains with constant gradients for the two-item inventory system with one-way substitution 
Domain $\Omega_{0}$ indicates the domain in which the demand of both product types can be satisfied by dedicated inventory $\left(0 \leq d_{1} \leq S_{1}\right.$ and $\left.0 \leq d_{2} \leq S_{2}\right)$. In domain $\Omega_{1}$, the demand of both product types is satisfied, but part of the demand of product 1 is rerouted to product $2\left(0 \leq d_{2} \leq S_{2}\right.$ and $\left.S_{1} \leq d_{1} \leq S_{1}+S_{2}-d_{2}\right)$. In domain $\Omega_{2}$, only the demand of product 1 is satisfied, part of the demand for product 2 is lost $(0 \leq$ $d_{1} \leq S_{1}$ and $\left.d_{2}>S_{2}\right)$. In domain $\Omega_{3}$, both items incur lost sales $\left(d_{1}>S_{1}\right.$ and $\left.d_{2}>S_{2}\right)$. In domain $\Omega_{4}$, the demand of product 2 is satisfied, while product 1 incurs lost sales $\left(d_{1}>S_{1}+S_{2}-d_{2}\right.$ and $0 \leq d_{2} \leq$ $\left.S_{2}\right)$.

The shadow prices for each of these 5 domains are derived in Appendix B, and summarized in Table 2 .

\begin{tabular}{|c|c|c|}
\hline Domain & $\boldsymbol{\lambda}_{\mathbf{1 j}}$ & $\boldsymbol{\lambda}_{\mathbf{2} \boldsymbol{j}}$ \\
\hline$\Omega_{0}$ & $c_{1}+h_{1}$ & $c_{2}+h_{2}$ \\
\hline$\Omega_{1}$ & $c_{1}-a+h_{2}$ & $c_{2}+h_{2}$ \\
\hline$\Omega_{2}$ & $c_{1}+h_{1}$ & $c_{2}-p_{2}$ \\
\hline$\Omega_{3}$ & $c_{1}-p_{1}$ & $c_{2}-p_{2}$ \\
\hline$\Omega_{4}$ & $c_{1}-p_{1}$ & $c_{2}-p_{1}+a$ \\
\hline
\end{tabular}

Table 2: Shadow prices for the 5 domains for the one-way substitution strategy

As, for any given $S_{1}$ and $S_{2}$, the shadow prices are constant in each demand domain, the expected shadow price of constraint $i$ can be calculated as:

$$
E\left[\lambda_{i}\right]=\sum_{j=0}^{4} \lambda_{i j} P\left(\Omega_{\mathrm{j}}\right)
$$

with $P\left(\Omega_{\mathrm{j}}\right)$ denoting the probability that the joint demand observation $\left(d_{1}, d_{2}\right)$ falls in domain $j^{5}$.

\footnotetext{
${ }^{5}$ Note that $P\left(\Omega_{j}\right)$ in fact depends on $\mathrm{S}_{1}$ and $\mathrm{S}_{2}$. To avoid the complex notation $P\left(\Omega_{j}\left(S_{1}, S_{2}\right)\right)$, we opt for the more compact notation $P\left(\Omega_{j}\right)$.
} 
Consequently, the first order derivatives of the objective function can be expressed conveniently as:

$$
\begin{aligned}
& \frac{\partial E[T C]}{\partial S_{1}}=\left(c_{1}+h_{1}\right) P\left(\Omega_{0}\right)+\left(c_{1}-a+h_{2}\right) P\left(\Omega_{1}\right)+\left(c_{1}+h_{1}\right) P\left(\Omega_{2}\right)+\left(c_{1}-p_{1}\right) P\left(\Omega_{3}\right)+ \\
& \left(c_{1}-p_{1}\right) P\left(\Omega_{4}\right) \\
& \frac{\partial E[T C]}{\partial S_{2}}=\left(c_{2}+h_{2}\right) P\left(\Omega_{0}\right)+\left(c_{2}+h_{2}\right) P\left(\Omega_{1}\right)+\left(c_{2}-p_{2}\right) P\left(\Omega_{2}\right)+\left(c_{2}-p_{2}\right) P\left(\Omega_{3}\right)+\left(c_{2}-p_{1}+\right. \\
& \text { a) } P\left(\Omega_{4}\right)
\end{aligned}
$$

Since $E[T C]$ is convex, we know that the optimal order-up-to levels $\left(S_{1}^{*}\right.$ and $\left.S_{2}^{*}\right)$ are unique. Setting both derivatives equal to zero yields (after some straightforward manipulations) the following set of optimal conditions:

$$
\begin{aligned}
& c_{1}+h_{1} P\left(\Omega_{0}^{S O *}+\Omega_{2}^{S O *}\right)=p_{1} P\left(\Omega_{3}^{S O *}+\Omega_{4}^{S O *}\right)+\left(a-h_{2}\right) P\left(\Omega_{1}^{S O *}\right) \\
& c_{2}+h_{2} P\left(\Omega_{0}^{S O *}+\Omega_{1}^{S O *}\right)=p_{2} P\left(\Omega_{2}^{S O *}+\Omega_{3}^{S O *}\right)+\left(p_{1}-a\right) P\left(\Omega_{4}^{S O *}\right)
\end{aligned}
$$

With $\Omega_{j}^{S O *}(j=0, \ldots, 4)$ demand domain $j$ that is determined by the optimal order up to levels $\left(S_{1}^{*}\right.$ and $\left.S_{2}^{*}\right)$ for the single period one-way substitution strategy.

The interpretation of these optimal conditions is quite intuitive. The left-hand side refers to the expected cost of raising the order-up-to level of product 1 (expression 3) and product 2 (expression 4) with one unit: this expected cost consists of the purchasing cost and the holding cost, which is only incurred in case inventory remains at the end of the period. The right-hand side refers to the expected benefit of such an increase: for item 1, it consists of the penalty cost that is avoided in case of unsatisfied demand, plus the benefit incurred by avoiding to reroute demand to product 2 (expression 3). For item 2 (expression 4 ), it consists of the avoided penalty cost and the benefit incurred by the possibility to reroute an additional unit of product 1 demand to product 2 (instead of incurring a shortage ). 
Combining expressions (3) and (4) with any continuous bivariate demand distribution (which can be mapped on Figure 3) enables us to derive the optimal order-up-to levels $\left(S_{1}^{*}\right.$ and $\left.S_{2}^{*}\right)$ : i.e., the order-up-to levels need to be set in such a way that the resulting $P\left(\Omega_{\mathrm{j}}\right)^{\prime}$ s cause expressions (3) and (4) to hold.

The strength of this newsvendor approach lies in its simplicity, and its graphical interpretation. For instance, the effect of allowing one-way substitution on the customer service level (CSL) of both products can be derived graphically. As illustrated in Figure 4a, the CSL of product 1 (in case a one-way substitution strategy is applied) equals the sum of $P\left(\Omega_{0}\right), P\left(\Omega_{1}\right)$ and $P\left(\Omega_{2}\right)$. Hence, the CSL of product 1 benefits from an increase in $S_{1}$ as well as $S_{2}$. As shown in Figure $4 \mathrm{~b}$, the CSL of product 2 equals the sum of $P\left(\Omega_{0}\right), P\left(\Omega_{1}\right)$ and $P\left(\Omega_{4}\right)$. Hence, the CSL of product 2 is only influenced by a change in $S_{2}$.

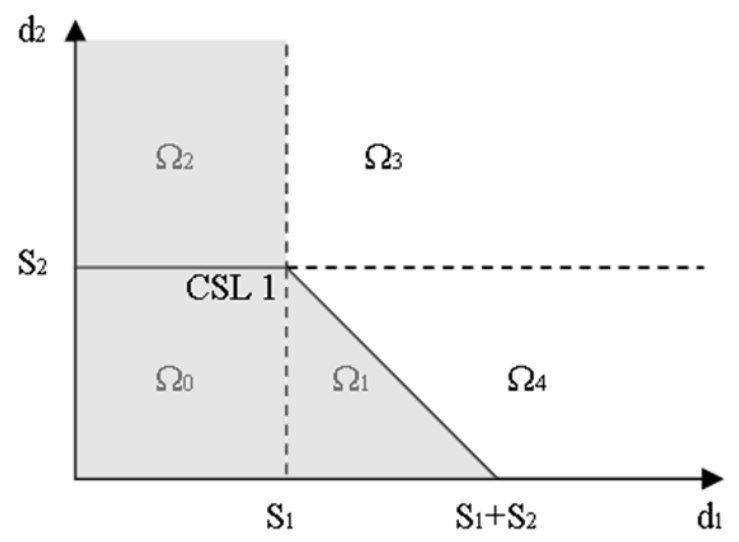

Fig. 4a: Customer service level of product 1

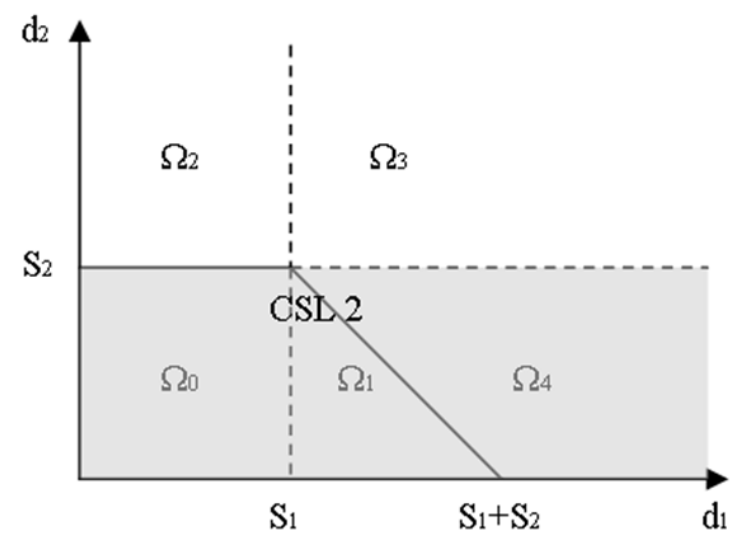

Fig. 4b: Customer service level of product 2

It is easily seen that for any given combination of order-up-to levels, allowing one-way substitution increases the CSL of product 1 by $P\left(\Omega_{1}\right)$ while the CSL of product 2 stays unchanged. 


\subsection{Borderline case: $S_{1}=0$}

In this section, we derive the optimal condition for a "borderline case" in which the order-up-to level of item 1 is reduced to zero. Note that this implies that all demand for item 1 is rerouted to the stock of item 2, which in fact coincides with full pooling. The purchasing cost $c_{1}$ turns out to play a pivotal role: we show that as long as the purchasing cost of product 1 stays below a threshold purchasing $\operatorname{cost} \overline{\mathrm{c}_{1}}$ the optimal order-up-to level of item 1 will be strictly positive; for purchasing costs above the threshold, the borderline case turns out to be optimal.

As the borderline case is an extreme case of the one-way substitution strategy (with $S_{1}=0$ ), this implies $P\left(\Omega_{0}\right)=P\left(\Omega_{2}\right)=0$, which reduces the demand space to only 3 domains $\Omega_{\mathrm{j}}(j=1,3$ and 4$)$ with constant shadow prices $\lambda_{\mathrm{ij}}$ (as in Figure 5).

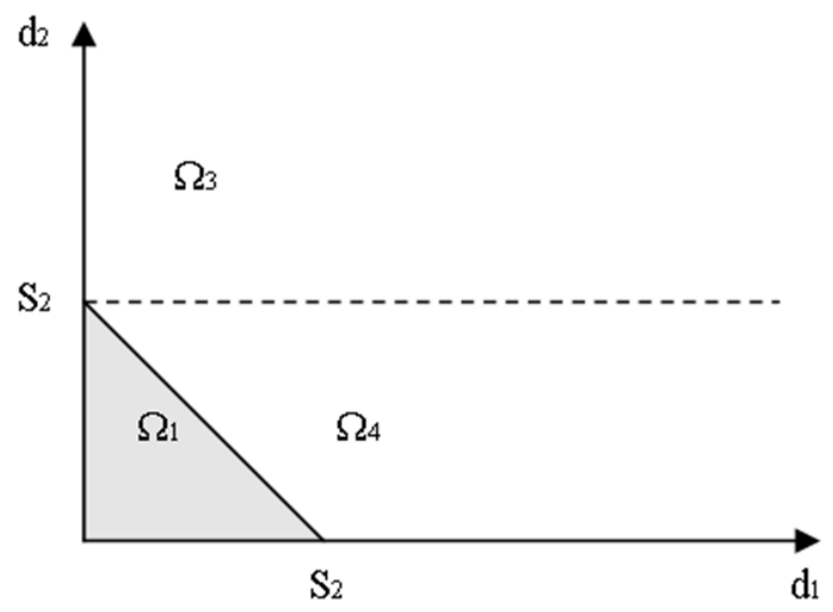

Fig. 5: Demand domains with constant gradients for the two-item inventory system with $S_{1}=0$

Since $S_{1}=0$ implies $x_{1}=0$, the expected total cost can be reformulated as:

$E[T C]=c_{2}\left(S_{2}\right)+h_{2} E\left[S_{2}-x_{2}-z\right]+p_{1} E\left[d_{1}-z\right]+p_{2} E\left[d_{2}-x_{2}\right]+a E[z]$ 
In order to derive the optimality condition, we need to calculate the expected shadow price. The shadow prices for the three demand domains can be derived from the following (second stage) linear programming model (LP2):

$\operatorname{Min} c_{2}\left(S_{2}\right)+h_{2}\left(S_{2}-x_{2}-z\right)+p_{1}\left(d_{1}-z\right)+p_{2}\left(d_{2}-x_{2}\right)+a z$

s.t.

$$
\begin{gathered}
x_{2}+z \leq S_{2} \\
z \leq d_{1} \\
x_{2} \leq d_{2} \\
x_{2} \text { and } z \geq 0
\end{gathered}
$$

The resulting shadow prices for each of the three domains are shown in Table 3.

\begin{tabular}{|c|c|}
\hline Domain & $\lambda_{2 j}$ \\
\hline$\Omega_{1}$ & $c_{2}+h_{2}$ \\
\hline$\Omega_{3}$ & $c_{2}-p_{2}$ \\
\hline$\Omega_{4}$ & $c_{2}-p_{1}+a$ \\
\hline
\end{tabular}

Table 3: Shadow prices for the 3 domains with full demand pooling

Since the expected shadow price is equal to the first-order derivative of $E[T C]$ to $S_{2}$ and $E[T C]$ is a convex function of $S_{2}$, we know that the optimal order-up-to level $\left(S_{2}^{*}\right)$ is unique, and is such that the following optimal condition holds:

$c_{2}+h_{2} P\left(\Omega_{1}^{S F *}\right)=p_{2} P\left(\Omega_{3}^{S F *}\right)+\left(p_{1}-a\right) P\left(\Omega_{4}^{S F *}\right)$ 
With $\Omega_{j}^{S F *}\left(j=1,3\right.$ and 4) demand domain $j$ that is determined by the optimal order up to level $S_{2}^{*}$ given that $S_{1}=0^{6}$. Note, expression (6) can be derived from expression (4) with $P\left(\Omega_{0}\right)=P\left(\Omega_{2}\right)=0$.

Combining expression (6) with expression (3) and $P\left(\Omega_{0}\right)=P\left(\Omega_{2}\right)=0$, we can obtain the following threshold purchasing $\operatorname{cost} \overline{\mathrm{c}_{1}}$ for item 1 :

$\overline{c_{1}}=\frac{c_{2} p_{1}}{p_{2}}+\left(a+\frac{h_{2} p_{1}}{p_{2}}-h_{2}\right) P\left(\Omega_{1}^{S F *}\right)+\left(\frac{a p_{1}}{p_{2}}-\frac{p_{1}{ }^{2}}{p_{2}}+p_{1}\right) P\left(\Omega_{4}^{S F *}\right)$

We can conclude that a strictly positive value for $S_{1}$ is optimal as long as $c_{1}<\overline{c_{1}}$. When $c_{1}>\overline{c_{1}}$, it is optimal to set $S_{1}=0$, resulting in the borderline case.

Strikingly, $\overline{\mathrm{c}_{1}}$ is independent of $h_{1}$. As such, the value of the "penalty" for leftover stock of product 1 at the end of the season does not play any role when deriving the threshold purchasing cost. Note that, when $p_{1}=p_{2}$, expression (7) reduces to:

$\overline{c_{1}}=c_{2}+a\left(P\left(\Omega_{1}^{S F *}\right)+P\left(\Omega_{4}^{S F *}\right)\right)=c_{2}+a P\left(d_{2} \leq S_{2}\right)$

As such, when the shortage costs for both products are equal, the borderline case can only be optimal when item 2 is actually cheaper than item 1 . Indeed, note that (8) implies that

$$
c_{1}-c_{2} \geq a P\left(d_{2} \leq S_{2}\right)
$$

where the right-hand side of the inequality is $\geq 0$.

\section{Multiperiod newsvendor model}

\subsection{One-way substitution}

The single period model can be extended to a multiperiod model ${ }^{7}$. The difference is that at the start of a period the amount ordered has to take into account the leftover inventory and backorders at the end of

\footnotetext{
${ }^{6}$ The superscript $\mathrm{F}$ refers to "full rerouting"; indeed, all demand for item 1 will be routinely rerouted to stock of item 2.
} 
previous period. For this reason, we introduce the subscripts $t$ and $t-1$ to refer to the current period and previous period, respectively. By analogy with expression (2), the expected total cost per period in the multiperiod setting can be determined as:

$E[T C]=c_{1}\left(S_{1}-E\left[S_{1}-x_{1, t-1}\right]+E\left[d_{1, t-1}-x_{1, t-1}-z_{t-1}\right]\right)+c_{2}\left(S_{2}-\right.$

$\left.E\left[S_{2}-x_{2, t-1}-z_{t-1}\right]+E\left[d_{2, t-1}-x_{2, t-1}\right]\right)+h_{1} E\left[S_{1}-x_{1, t}\right]+h_{2} E\left[S_{2}-x_{2, t}-z_{t}\right]+$

$p_{1} E\left[d_{1, t}-x_{1, t}-z_{t}\right]+p_{2} E\left[d_{2, t}-x_{2, t}\right]+a E\left[z_{t}\right]$

The difference between expressions (9) and (2) lies in the amount ordered for both products. The expected amount ordered of product $i$ is equal to the order-up-to level minus the expected starting inventory plus the expected backorders. Additionally, the expected total cost depends on demand and allocation decisions of two periods (period $t-1$ and period $t$ ). As mentioned in Section 2, the fact that the cost parameters and demand distribution do not change over time implies that the optimal order up to levels will be time-invariant too.

When determining the optimal allocation decision (second-stage decision) for period $t\left(x_{1, t}^{*}, x_{2, t}^{*}\right.$ and $\left.z_{t}^{*}\right)$, given an order-up-to level $S_{1}$ and $S_{2}$, the optimal allocation decisions of the previous period have been taken $\left(x_{1, t-1}^{*}, x_{2, t-1}^{*}\right.$ and $\left.z_{t-1}^{*}\right)$, and the demands of the previous and current periods are known $\left(d_{1, t-1}\right.$, $d_{2, t-1}, d_{1, t}$ and $\left.d_{2, t}\right)$. The optimal allocation decision in period $\mathrm{t}$ corresponds to the solution of the following linear programming model (LP3):

Min $c_{1}\left(d_{1, t-1}-z_{t-1}^{*}\right)+c_{2}\left(d_{2, t-1}+z_{t-1}^{*}\right)+h_{1}\left(S_{1}-x_{1, t}\right)+h_{2}\left(S_{2}-x_{2, t}-z_{t}\right)$

$+p_{1}\left(d_{1, t}-x_{1, t}-z_{t}\right)+p_{2}\left(d_{2, t}-x_{2, t}\right)+a\left(z_{t}\right)$

s.t.

$$
\begin{gathered}
x_{1, t} \leq S_{1} \\
x_{2, t}+z_{t} \leq S_{2} \\
x_{1, t}+z_{t} \leq d_{1, t}
\end{gathered}
$$

\footnotetext{
${ }^{7}$ As mentioned in section 2, Assumption 5 is not required in the multiperiod setting.
} 


$$
\begin{gathered}
x_{2, t} \leq d_{2, t} \\
x_{1, t}, x_{2, t} \text { and } z_{t} \geq 0
\end{gathered}
$$

Note that the value of $z_{t-1}^{*}$ depends both on the order-up-to levels $\left(S_{1}\right.$ and $\left.S_{2}\right)$ and on the demand of period $t-1$. Consequently, to determine the expected shadow prices of $S_{1}$ and $S_{2}$, we need to take into account the probability that demand falls in a demand domain $\Omega_{j}$ not only for the current period's demand, but also for the previous period's demand (as this impacts $z_{t-1}^{*}$ ). Figure 6a illustrates the different demand domains for period $t-1$ with the associated $z_{t-1}^{*}$ values. In Figure $6 \mathrm{~b}$ the demand domains for period $t$ are presented ${ }^{8}$.

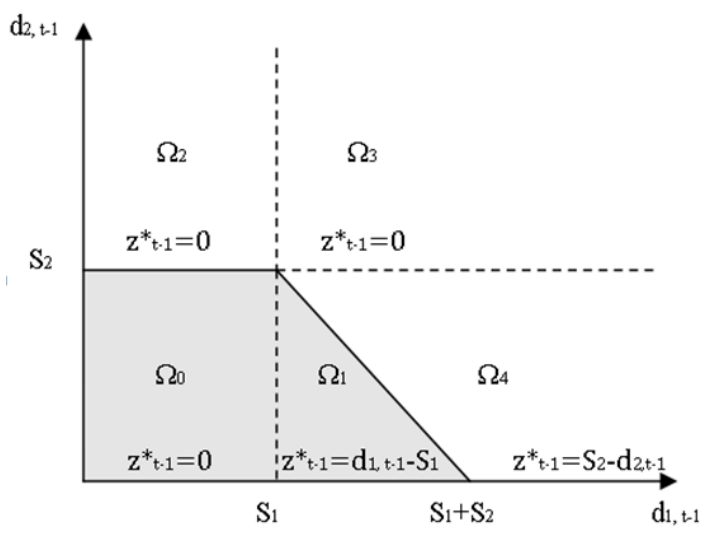

Fig. 6a: Demand domains and associated

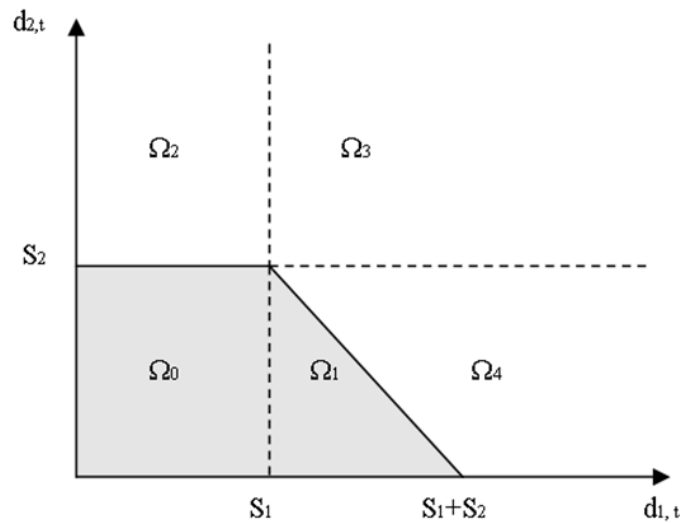

Fig. 6b: Demand domains for period $t$ $\mathrm{z}_{\mathrm{t}-1}$ values for period $t-1$

The optimal allocation decisions $\left(x_{1, t}^{*}, x_{2, t}^{*}\right.$ and $\left.z_{t}^{*}\right)$ can now be determined, and shadow prices can be derived. Let $\lambda_{i j k}$ denote the shadow price of constraint $i$ when the demand in the previous period falls in domain $j$ and the demand of the current period falls in domain $k$. Table 4 summarizes $\lambda_{i j k}(i=1,2)$ for each combination of demand domains.

\footnotetext{
${ }^{8} \mathrm{We}$ assume that the demands in successive periods are independent.
} 


\begin{tabular}{|c|c|c|c|c|c|c|c|}
\hline $\begin{array}{l}\text { Domain } \\
\text { period } t\end{array}$ & $x_{1, t}^{*}$ & $x_{2, t}^{*}$ & $z_{t}^{*}$ & $\begin{array}{c}\text { Domain } \\
\text { period } t-1\end{array}$ & $z_{t-1}^{*}$ & $\lambda_{1 j k}$ & $\lambda_{2 j k}$ \\
\hline \multirow{5}{*}{$\Omega_{0}$} & \multirow{5}{*}{$d_{1, t}$} & \multirow{5}{*}{$d_{2, t}$} & \multirow{5}{*}{0} & $\Omega_{0}$ & 0 & $h_{1}$ & $h_{2}$ \\
\hline & & & & $\Omega_{1}$ & $d_{1, t-1}-S_{1}$ & $c_{1}-c_{2}+h_{1}$ & $h_{2}$ \\
\hline & & & & $\Omega_{2}$ & 0 & $h_{1}$ & $h_{2}$ \\
\hline & & & & $\Omega_{3}$ & 0 & $h_{1}$ & $h_{2}$ \\
\hline & & & & $\Omega_{4}$ & $S_{2}-d_{2, t-1}$ & $h_{1}$ & $-c_{1}+c_{2}+h_{2}$ \\
\hline \multirow{5}{*}{$\Omega_{1}$} & \multirow{5}{*}{$S_{1}$} & \multirow{5}{*}{$d_{2, t}$} & \multirow{5}{*}{$\begin{array}{l}d_{1, t} \\
-S_{1}\end{array}$} & $\Omega_{0}$ & 0 & $h_{2}-a$ & $h_{2}$ \\
\hline & & & & $\Omega_{1}$ & $d_{1, t-1}-S_{1}$ & $c_{1}-c_{2}+h_{2}-a$ & $h_{2}$ \\
\hline & & & & $\Omega_{2}$ & 0 & $h_{2}-a$ & $h_{2}$ \\
\hline & & & & $\Omega_{3}$ & 0 & $h_{2}-a$ & $h_{2}$ \\
\hline & & & & $\Omega_{4}$ & $S_{2}-d_{2, t-1}$ & $h_{2}-a$ & $-c_{1}+c_{2}+h_{2}$ \\
\hline \multirow{5}{*}{$\Omega_{2}$} & \multirow{5}{*}{$d_{1, t}$} & \multirow{5}{*}{$S_{2}$} & \multirow{5}{*}{0} & $\Omega_{0}$ & 0 & $h_{1}$ & $-p_{2}$ \\
\hline & & & & $\Omega_{1}$ & $d_{1, t-1}-S_{1}$ & $c_{1}-c_{2}+h_{1}$ & $-p_{2}$ \\
\hline & & & & $\Omega_{2}$ & 0 & $h_{1}$ & $-p_{2}$ \\
\hline & & & & $\Omega_{3}$ & 0 & $h_{1}$ & $-p_{2}$ \\
\hline & & & & $\Omega_{4}$ & $S_{2}-d_{2, t-1}$ & $h_{1}$ & $-c_{1}+c_{2}-p_{2}$ \\
\hline \multirow{5}{*}{$\Omega_{3}$} & \multirow{5}{*}{$S_{1}$} & \multirow{5}{*}{$S_{2}$} & \multirow{5}{*}{0} & $\Omega_{0}$ & 0 & $-p_{1}$ & $-p_{2}$ \\
\hline & & & & $\Omega_{1}$ & $d_{1, t-1}-S_{1}$ & $c_{1}-c_{2}-p_{1}$ & $-p_{2}$ \\
\hline & & & & $\Omega_{2}$ & 0 & $-p_{1}$ & $-p_{2}$ \\
\hline & & & & $\Omega_{3}$ & 0 & $-p_{1}$ & $-p_{2}$ \\
\hline & & & & $\Omega_{4}$ & $S_{2}-d_{2, t-1}$ & $-p_{1}$ & $-c_{1}+c_{2}-p_{2}$ \\
\hline \multirow{5}{*}{$\Omega_{4}$} & \multirow{5}{*}{$S_{1}$} & \multirow{5}{*}{$d_{2, t}$} & \multirow{5}{*}{$\begin{array}{l}S_{2} \\
-d_{2, t}\end{array}$} & $\Omega_{0}$ & 0 & $-p_{1}$ & $-p_{1}+a$ \\
\hline & & & & $\Omega_{1}$ & $d_{1, t-1}-S_{1}$ & $c_{1}-c_{2}-p_{1}$ & $-p_{1}+a$ \\
\hline & & & & $\Omega_{2}$ & 0 & $-p_{1}$ & $-p_{1}+a$ \\
\hline & & & & $\Omega_{3}$ & 0 & $-p_{1}$ & $-p_{1}+a$ \\
\hline & & & & $\Omega_{4}$ & $S_{2}-d_{2, t-1}$ & $-p_{1}$ & $-c_{1}+c_{2}-p_{1}+a$ \\
\hline
\end{tabular}

Table 4: Shadow prices for the multiperiod one-way substitution strategy

For the single-period setting, we already proved that the objective function is convex in $S_{1}$ and $S_{2}$. The objective function of (LP3) is similar to the one observed in (LP1), except for the $z_{t-1}^{*}$ values appearing in the first two terms. However, as $z_{t-1}^{*}$ is equal to $0, d_{1, t-1}-S_{1}$ or $S_{2}-d_{2, t-1}$ (see Table 4: the actual value depends on the demand domain), the first two terms remain convex in $S_{1}$ and $S_{2}$. Consequently, the objective function of (LP2) and (by extension) the function $E[T C]$ in expression (9) remain convex in $S_{1}$ and $S_{2}$. 
The expected shadow prices or first-order derivatives can be calculated as $\partial E[T C] / \partial S_{i}=E\left[\lambda_{i}\right]=$ $\sum_{j=0}^{4} \sum_{k=0}^{4} \lambda_{i j k} P\left(\Omega_{j}\right) P\left(\Omega_{k}\right)(i=1,2):$

$$
\begin{aligned}
& \frac{\partial E[T C]}{\partial S_{1}}=\left[h_{1}\left(1-P\left(\Omega_{1}\right)\right)+\left(c_{1}-c_{2}+h_{1}\right) P\left(\Omega_{1}\right)\right]\left(P\left(\Omega_{0}\right)+P\left(\Omega_{2}\right)\right)+\left[\left(h_{2}-a\right)\left(1-P\left(\Omega_{1}\right)\right)+\right. \\
& \left.\left(c_{1}-c_{2}+h_{2}-a\right) P\left(\Omega_{1}\right)\right] P\left(\Omega_{1}\right)+\left[-p_{1}\left(1-P\left(\Omega_{1}\right)\right)+\left(c_{1}-c_{2}-p_{1}\right) P\left(\Omega_{1}\right)\right]\left(P\left(\Omega_{3}\right)+P\left(\Omega_{4}\right)\right)
\end{aligned}
$$$$
\frac{\partial E[T C]}{\partial S_{2}}=\left[h_{2}\left(1-P\left(\Omega_{4}\right)\right)+\left(-c_{1}+c_{2}+h_{2}\right) P\left(\Omega_{4}\right)\right]\left(P\left(\Omega_{0}\right)+P\left(\Omega_{1}\right)\right)+\left[-p_{2}\left(1-P\left(\Omega_{4}\right)\right)+\right.
$$$$
\left.\left(-c_{1}+c_{2}-p_{2}\right) P\left(\Omega_{4}\right)\right]\left(P\left(\Omega_{2}\right)+P\left(\Omega_{3}\right)\right)+\left[\left(-p_{1}+a\right)\left(1-P\left(\Omega_{4}\right)\right)+\left(-c_{1}+c_{2}-p_{1}+\right.\right.
$$

a) $\left.P\left(\Omega_{4}\right)\right] P\left(\Omega_{4}\right)$

Since $E[T C]$ is convex in $S_{1}$ and $S_{2}, \frac{\partial E[T C]}{\partial S_{1}}=\frac{\partial E[T C]}{\partial S_{2}}=0$ are 2 necessary and sufficient conditions to determine the optimal and unique order-up-to levels $\left(S_{1}^{*}\right.$ and $\left.S_{2}^{*}\right)$. The optimality conditions are:

$\left(c_{1}-c_{2}\right) P\left(\Omega_{1}^{M O *}\right)+h_{1} P\left(\Omega_{0}^{M O *}+\Omega_{2}^{M O *}\right)=p_{1} P\left(\Omega_{3}^{M O *}+\Omega_{4}^{M O *}\right)+\left(a-h_{2}\right) P\left(\Omega_{1}^{M O *}\right)$

$\left(c_{2}-c_{1}\right) P\left(\Omega_{4}^{M O *}\right)+h_{2} P\left(\Omega_{0}^{M O *}+\Omega_{1}^{M O *}\right)=p_{2} P\left(\Omega_{2}^{M O *}+\Omega_{3}^{M O *}\right)+\left(p_{1}-a\right) P\left(\Omega_{4}^{M O *}\right)$

with $\Omega_{j}^{M O *}(j=0, \ldots, 4)$ demand domain $j$ that is determined by the optimal order up to levels $\left(S_{1}^{*}\right.$ and $\left.S_{2}^{*}\right)$ for the multiperiod one-way substitution strategy.

Note that, except for the first terms, expressions (10) and (11) equal the optimality conditions for the single-period setting (expressions (3) and (4)). The left-hand side refers to the expected cost of raising the order-up-to level of product 1 (expression 10) and product 2 (expression 11) with one unit, while the right-hand side refers to the expected benefit of such a change. The first term of expressions (10) and (11) can be explained by examining the order quantities in period $t$ conditional on the prevailing demand domain in period $t-1$. These are shown in Table 5. 


\begin{tabular}{|c|c|c|}
\hline $\begin{array}{c}\text { Domain in } \\
\text { period } \boldsymbol{t}-\mathbf{1}\end{array}$ & Order quantity in period $\boldsymbol{t}$ product $\mathbf{1}$ & Order quantity in period $\boldsymbol{t}$ product 2 \\
\hline$\Omega_{0}$ & $d_{1, t-1}$ & $d_{2, t-1}$ \\
\hline$\Omega_{1}$ & $d_{1, t-1}-z_{t-1}=S_{1}$ & $d_{2, t-1}+z_{t-1}=d_{2, t-1}+d_{1, t-1}-S_{1}$ \\
\hline$\Omega_{2}$ & $d_{1, t-1}$ & $d_{2, t-1}$ \\
\hline$\Omega_{3}$ & $d_{1, t-1}$ & $d_{2, t-1}$ \\
\hline$\Omega_{4}$ & $d_{1, t-1}-z_{t-1}=d_{1, t-1}-S_{2}+d_{2, t-1}$ & $d_{2, t-1}+z_{t-1}=S_{2}$ \\
\hline
\end{tabular}

Table 5: The order quantity for both products, conditional on the demand domain in period $t-1$

From Table 5, we can derive that an increase in $S_{1}$ only impacts the order quantities when the previous period's demand falls in domain $\Omega_{1}$ : in this case, the order quantity of product 1 increases with one unit while the order quantity of product 2 decreases with one unit (explaining the $\left(c_{1}-c_{2}\right)$ factor in the first term of expression (10)). Similarly, an increase in $S_{2}$ only impacts the order quantities when the previous period's demand falls in domain $\Omega_{4}$, leading to a unit decrease in the order quantity of product 1 and a unit increase in the order quantity of product 2 (as evident from the first term in expression (11)).

We can reformulate expressions (10) and (11) as:

$h_{1} P\left(\Omega_{0}^{M O *}+\Omega_{2}^{M O *}\right)=p_{1} P\left(\Omega_{3}^{M O *}+\Omega_{4}^{M O *}\right)+\left(a-h_{2}-c_{1}+c_{2}\right) P\left(\Omega_{1}^{M O *}\right)$

$h_{2} P\left(\Omega_{0}^{M O *}+\Omega_{1}^{M O *}\right)=p_{2} P\left(\Omega_{2}^{M O *}+\Omega_{3}^{M O *}\right)+\left(p_{1}-a-c_{2}+c_{1}\right) P\left(\Omega_{4}^{M O *}\right)$

From these expressions we can clearly see that changing purchasing costs and adjustment costs in such a way that the flexibility cost is kept unchanged does not affect the optimal order-up-to values, since the coefficients in both conditions will remain unchanged. 


\subsection{Borderline case: $S_{1}=0$}

In this section, we derive the optimal condition for the multiperiod case with $S_{1}=0$. Note that for the multiperiod case this implies that all demand for item 1 is rerouted to the stock of item 2; only when backorders are incurred for item 1, a replenishment order for item 1 is triggered. Though this borderline case is likely to be irrelevant in practice (it is rather counterintuitive to routinely satisfy demand for product 1 by means of type 2 items, while meeting backorders by means of type 1 items), it may turn out to be the mathematically optimal solution. In what follows, we determine the threshold purchasing cost $\overline{c_{1}}$ : when $c_{1}$ exceeds this threshold, the borderline case turns out to be mathematically optimal.

As $S_{1}=0$, which implies $P\left(\Omega_{0}\right)=P\left(\Omega_{2}\right)=0$, the demand space for period $t-1$ and period $t$ reduces to only 3 domains $\Omega_{\mathrm{j}}(j=1,3$ and 4$)$. Figure 7a illustrates the different demand domains for period $t-1$ with the associated $z_{t-1}^{*}$ values. In Figure $7 \mathrm{~b}$ the demand domains for period $t$ are presented.

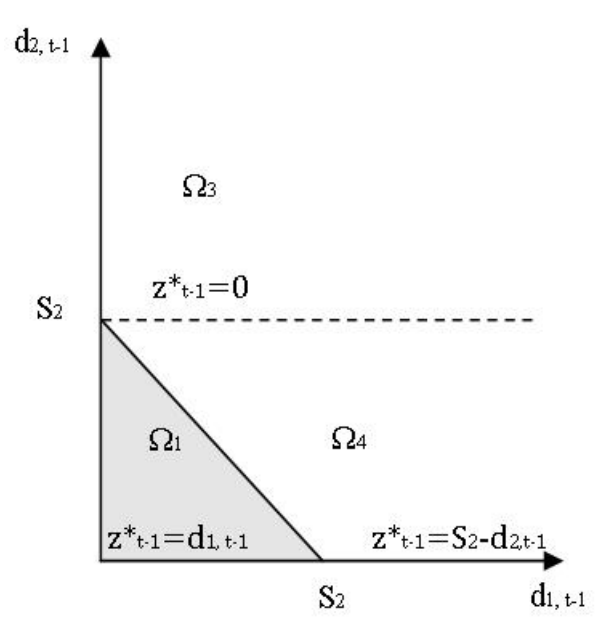

Fig. 7a: Demand domains and associated

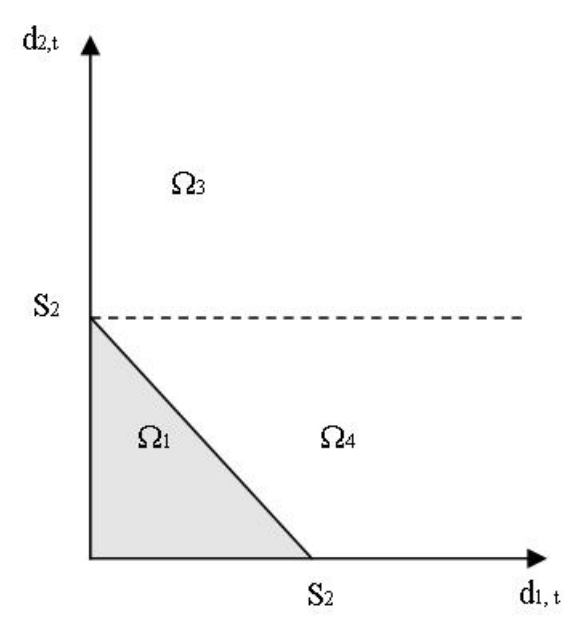

Fig. 7b: Demand domains for period $t$

$$
\mathrm{z}_{\mathrm{t}-1} \text { values for period } t-1
$$

Since $S_{1}=0$ implies $x_{1, t-1}=x_{1, t}=0$, the expected total cost can be reformulated as:

$E[T C]=c_{1}\left(E\left[d_{1, t-1}-z_{t-1}\right]\right)+c_{2}\left(S_{2}-E\left[S_{2}-x_{2, t-1}-z_{t-1}\right]+E\left[d_{2, t-1}-x_{2, t-1}\right]\right)$ 
$+h_{2} E\left[S_{2}-x_{2, t}-z_{t}\right]+p_{1} E\left[d_{1, t}-z_{t}\right]+p_{2} E\left[d_{2, t}-x_{2, t}\right]+a E\left[z_{t}\right]$

The shadow prices in each of the three demand domains can be derived from the following (second stage) linear programming model (LP4):

Min $c_{1}\left(d_{1, t-1}-z_{t-1}^{*}\right)+c_{2}\left(d_{2, t-1}+z_{t-1}^{*}\right)+h_{2}\left(S_{2}-x_{2, t}-z_{t}\right)+p_{1}\left(d_{1, t}-z_{t}\right)+p_{2}\left(d_{2, t}-x_{2, t}\right)+$ $a\left(z_{t}\right)$

s.t.

$$
\begin{gathered}
x_{2, t}+z_{t} \leq S_{2} \\
z_{t} \leq d_{1, t} \\
x_{2, t} \leq d_{2, t} \\
x_{2, t} \text { and } z_{t} \geq 0
\end{gathered}
$$

\begin{tabular}{|c|c|c|c|c|c|}
\hline $\begin{array}{l}\text { Domain } \\
\text { period } t\end{array}$ & $x_{2, t}^{*}$ & $\mathbf{Z}_{t}^{*}$ & $\begin{array}{c}\text { Domain } \\
\text { period } t-1\end{array}$ & $z_{t-1}^{*}$ & $\lambda_{2 j k}$ \\
\hline \multirow{3}{*}{$\Omega_{1}$} & \multirow{3}{*}{$d_{2, t}$} & \multirow{3}{*}{$d_{1, t}$} & $\Omega_{1}$ & $d_{1, t-1}$ & $h_{2}$ \\
\hline & & & $\Omega_{3}$ & 0 & $h_{2}$ \\
\hline & & & $\Omega_{4}$ & $S_{2}-d_{2, t-1}$ & $-c_{1}+c_{2}+h_{2}$ \\
\hline \multirow{3}{*}{$\Omega_{3}$} & \multirow{3}{*}{$S_{2}$} & \multirow{3}{*}{0} & $\Omega_{1}$ & $d_{1, t-1}$ & $-p_{2}$ \\
\hline & & & $\Omega_{3}$ & 0 & $-p_{2}$ \\
\hline & & & $\Omega_{4}$ & $S_{2}-d_{2, t-1}$ & $-c_{1}+c_{2}-p_{2}$ \\
\hline \multirow{3}{*}{$\Omega_{4}$} & \multirow{3}{*}{$d_{2, t}$} & \multirow{3}{*}{$S_{2}-d_{2, t}$} & $\Omega_{1}$ & $d_{1, t-1}$ & $-p_{1}+a$ \\
\hline & & & $\Omega_{3}$ & 0 & $-p_{1}+a$ \\
\hline & & & $\Omega_{4}$ & $S_{2}-d_{2, t-1}$ & $-c_{1}+c_{2}-p_{1}+a$ \\
\hline
\end{tabular}

The resulting shadow prices for each combination of demand domains in period $t-1$ and $t$ are shown in Table 6.

Table 6: Shadow prices for the 3 domains with $S_{1}=0$ 
As in the single period setting, $E[T C]$ is a convex function of $S_{2}$ and consequently the optimal order-up-to level $\left(S_{2}^{*}\right)$ is unique and is determined by the first-order derivative of $E[T C]$ to $S_{2}$. The optimality condition is:

$\left(c_{2}-c_{1}\right) P\left(\Omega_{4}^{M F *}\right)+h_{2} P\left(\Omega_{1}^{M F *}\right)=p_{2} P\left(\Omega_{3}^{M F *}\right)+\left(p_{1}-a\right) P\left(\Omega_{4}^{M F *}\right)$

With $\Omega_{j}^{M F *}(j=1,3$ and 4$)$ demand domain $j$ as determined by the optimal order up to level $S_{2}^{*}$ for the multiperiod one-way substitution strategy with $S_{1}=0$.

Combining expression (10) with $P\left(\Omega_{0}\right)=P\left(\Omega_{2}\right)=0$, we know that the borderline case will be optimal if:

$$
c_{1} \geq p_{1}\left(\left(P\left(\Omega_{3}^{M F *}\right)+P\left(\Omega_{4}^{M F *}\right)\right) / P\left(\Omega_{1}^{M F *}\right)\right)+a-h_{2}+c_{2}
$$

Using the information in expression (13), this can be rewritten as:

$$
c_{1} \geq \frac{p_{1}}{p_{2}}\left[\left(c_{2}-c_{1}+a-p_{1}+p_{2}\right) \frac{P\left(\Omega_{4}^{*}\right)}{P\left(\Omega_{1}^{*}\right)}+h_{2}\right]+a-h_{2}+c_{2}
$$

which results in the following threshold purchasing cost for product 1 :

$\overline{\mathrm{c}_{1}}=\left(\frac{p_{1}}{p_{2}}\left[\left(c_{2}+a-p_{1}+p_{2}\right) \frac{P\left(\Omega_{4}^{M F *}\right)}{P\left(\Omega_{1}^{M F *}\right)}+h_{2}\right]+a-h_{2}+c_{2}\right) /\left(1+\frac{p_{1} P\left(\Omega_{4}^{M F *}\right)}{p_{2} P\left(\Omega_{1}^{M F *}\right)}\right)$

As in the single period case, $\overline{c_{1}}$ is independent of $h_{1}$. Note that, when $p_{1}=p_{2}$, expression (14) reduces to:

$\overline{c_{1}}=c_{2}+a$

As such, when $p_{1}=p_{2}$, the borderline case can only be optimal when the flexibility cost is negative (which violates our assumption 1). Consequently, when $p_{1}=p_{2}$, the borderline case can never be optimal in our setting. In Appendix C, we show (by extension) that the same conclusion holds for all $p_{1}$ values in the interval $\left[p_{2}, \frac{h_{2} P\left(\Omega_{1}^{M F *}\right)}{P\left(\Omega_{4}^{M F *}\right)}\right]$. 


\section{Conclusion}

This paper discussed an intuitive and insightful approach to determine optimal order-up-to levels in inventory systems with one-way substitution. Optimality conditions can be derived both for a single period and a multi period setting: essentially, it is shown that order-up-to levels should be set in such a way that the two-dimensional demand distribution "maps" onto the demand domains in such a way that conditions (3)-(4) hold for the single period setting, and conditions (10)-(11) hold for the multiperiod setting. The optimal order-up-to levels are shown to be unique, in both settings. In addition, we have shown that (for both single-period and multiperiod settings) reducing the order-up-to level of the inflexible item to 0 can only be optimal when the purchasing cost of this item exceeds a given threshold.

\section{References}

[1] Bassok Y., R. Anupindi and R. Akella (1999). Single-period multiproduct inventory models with substitution. Operations Research 47, 632-642.

[2] Chopra and Meindl (2007). Supply Chain Management: Strategy, planning \& Operation, third edition. Pearson Prentice Hall, New Jersey.

[3] Deflem Y. and I. Van Nieuwenhuyse (2011). A Discrete Time Markov Chain Model for a Periodic Inventory System with One-Way Substitution. Leuven. Unpublished technical report.

[4] Hale W., D. Pyke and N. Rudi (2001). An assemble-to-order system with component substitution. Working Paper, Tuck School of Business.

[5] Harrison J.M. and Van Mieghem J.A. (1999). Multi-resource investment strategies: Operational hedging under demand uncertainty. European Journal of Operational Research 113, 17-29. 
[6] Herer Y.T., M. Tzur and E. Yücesan (2006). The multilocation transshipment problem. IIE Transactions 38, 185-200.

[7] Heyman D.P. and M.J. Sobel (1990). Stochastic models. Elsevier Science Publisher, NorthHolland.

[8] Khouja M., A. Mehrez and G. Rabinowitz (1996). A two-item newsboy problem with substitution. International Journal of production Economics 44, 267-275.

[9] Robinson L.W. (1990). Optimal and approximate policies in multiperiod, multilocation inventory models with transshipments. Operations research 38, 278-295.

[10] Rutten and Bertrand (1998). Balancing stocks, flexible recipe costs and high service level requirements in a batch process industry: A study of a small scale model. European Journal of Operational Research 110, 626-642.

[11] Tibben-Lembke R.S. and Y. Bassok (2005). An inventory model for delayed customization: A hybrid approach. European Journal of Operational Research 165, 748-764.

[12] Van Mieghem J. A. (1998). Investment Strategies for Flexible Resources. Management Science 44, 1071-1078.

[13] Van Mieghem J. A. (2008), Operations Strategy: Principles and Practice, Dynamic Ideas, Belmont.

[14] Wets R.J.B. (1966). Programming under uncertainty: the equivalent convex program. SIAM Journal on Applied Mathematics 14, 89-105. 


\section{Appendix A}

In this section we prove that the objective function of (LP1) (i.e. $\left.\operatorname{TC}\left(S_{1}, S_{2}, d_{1}, d_{2}\right)\right)$ is convex in $S_{1}$ and $S_{2}$ for a given $d_{1}$ and $d_{2}$. First, note that the objective function can be rewritten as follows:

$$
\begin{aligned}
T C\left(S_{1}, S_{2}, d_{1}, d_{2}\right) & =\left(c_{1}+h_{1}\right) S_{1}+\left(c_{2}+h_{2}\right) S_{2}+p_{1} d_{1}+p_{2} d_{2}-\left(h_{1}+p_{1}\right) x_{1}-\left(h_{2}+p_{2}\right)\left(x_{2}\right) \\
+ & \left(a-h_{2}-p_{1}\right) z
\end{aligned}
$$

The first two terms boil down to linear functions of $S_{1}$ and $S_{2}$ while the third and fourth term are constants (independent of $S_{1}$ and $S_{2}$ ). Consequently, it remains to be proven that the sum of the last three terms is convex in $S_{1}$ and $S_{2}$. The proof is analogous to the one discussed in [14]. It is sufficient to prove that

$$
k(\boldsymbol{b})=\left\{\min _{\mathbf{y}} \boldsymbol{f} \boldsymbol{y} \mid \boldsymbol{A} \boldsymbol{y} \leq \boldsymbol{b}, \boldsymbol{y} \geq \mathbf{0}\right\}
$$

is convex in $\boldsymbol{b}$ with (in our case)

$$
\boldsymbol{f}=\left[\begin{array}{lll}
-h_{1}-p_{1} & -h_{2}-p_{2} & -h_{2}-p_{1}+a
\end{array}\right], \boldsymbol{y}=\left[\begin{array}{c}
x_{1} \\
x_{2} \\
z
\end{array}\right], \boldsymbol{A}=\left[\begin{array}{lll}
1 & 0 & 0 \\
0 & 1 & 1 \\
1 & 0 & 1 \\
0 & 1 & 0
\end{array}\right] \text { and } \boldsymbol{b}=\left[\begin{array}{l}
S_{1} \\
S_{2} \\
d_{1} \\
d_{2}
\end{array}\right]
$$

Consider $\boldsymbol{b}_{\boldsymbol{\theta}}, \boldsymbol{b}_{1}$ and $\boldsymbol{b}_{\boldsymbol{\theta}}=\boldsymbol{\theta} \boldsymbol{b}_{\mathbf{0}}+(\boldsymbol{1}-\boldsymbol{\theta}) \boldsymbol{b}_{\mathbf{1}}$ with $0 \leq \theta \leq 1$.

Assume that $\boldsymbol{y}_{\mathbf{0}}, \boldsymbol{y}_{\mathbf{1}}$ and $\boldsymbol{y}_{\boldsymbol{\theta}}$ are the optimal solutions of (LPA.1) for $\boldsymbol{b}_{\boldsymbol{\theta}}, \boldsymbol{b}_{\boldsymbol{1}}$ and $\boldsymbol{b}_{\boldsymbol{\theta}}$ respectively, so

$$
f y_{0}=k\left(b_{0}\right), f y_{1}=k\left(b_{1}\right), f y_{\theta}=k\left(b_{\theta}\right)
$$

$\overline{\boldsymbol{y}}_{\boldsymbol{\theta}}=\boldsymbol{\theta} \boldsymbol{y}_{\mathbf{0}}+(\mathbf{1}-\boldsymbol{\theta}) \boldsymbol{y}_{\mathbf{1}}$ is then a feasible solution of (LPA.1) with $\boldsymbol{b}=\boldsymbol{b}_{\boldsymbol{\theta}}$ since

$$
A \bar{y}_{\theta}=A \theta y_{0}+A(1-\theta) y_{1}=\theta A y_{0}+(1-\theta) A y_{1} \leq \theta b_{0}+(1-\theta) b_{1}=b_{\theta}
$$

Note, however, that $\overline{\boldsymbol{y}}_{\boldsymbol{\theta}}$ is not necessary an optimal solution of (LPA.1) with $\boldsymbol{b}=\boldsymbol{b}_{\boldsymbol{\theta}}$. We then have 


$$
\boldsymbol{f} \overline{\boldsymbol{y}}_{\boldsymbol{\theta}} \geq \boldsymbol{f} \boldsymbol{y}_{\boldsymbol{\theta}}=k\left(\boldsymbol{b}_{\boldsymbol{\theta}}\right)
$$

which implies

$$
\boldsymbol{\theta} k\left(\boldsymbol{b}_{\mathbf{0}}\right)+(\mathbf{1}-\boldsymbol{\theta}) k\left(\boldsymbol{b}_{\mathbf{1}}\right) \geq k\left(\boldsymbol{\theta} \boldsymbol{b}_{\mathbf{0}}+(\mathbf{1}-\boldsymbol{\theta}) \boldsymbol{b}_{\mathbf{1}}\right)
$$

This proves that $k(\boldsymbol{b})$ is indeed convex in $\boldsymbol{b}$. 


\section{Appendix B}

The shadow prices are calculated for each of the 5 domains separately. For a given $d_{1}, d_{2}, S_{1}$ and $S_{2}$ the optimal allocation decisions $\left(x_{1}^{*}, x_{2}^{*}\right.$ and $\left.z^{*}\right)$ can be derived for each domain. Rewriting the resulting objective function allows to derive the shadow prices in a straightforward way. Table B.1 summarizes the results for each demand domain.

\begin{tabular}{|c|c|c|c|c|c|c|}
\hline Domain & $\boldsymbol{x}_{\mathbf{1}}^{*}$ & $\boldsymbol{x}_{\mathbf{2}}^{*}$ & $\boldsymbol{z}^{*}$ & Objective function & $\boldsymbol{\lambda}_{\boldsymbol{j} \boldsymbol{j}}$ & $\boldsymbol{\lambda}_{\mathbf{2} \boldsymbol{j}}$ \\
\hline$\Omega_{0}$ & $d_{1}$ & $d_{2}$ & 0 & $c_{1}\left(S_{1}\right)+c_{2}\left(S_{2}\right)+h_{1}\left(S_{1}-d_{1}\right)+h_{2}\left(S_{2}-d_{2}\right)$ & $c_{1}+h_{1}$ & $c_{2}+h_{2}$ \\
\hline$\Omega_{1}$ & $S_{1}$ & $d_{2}$ & $d_{1}-S_{1}$ & $c_{1}\left(S_{1}\right)+c_{2}\left(S_{2}\right)+h_{2}\left(S_{2}-d_{2}-\left(d_{1}-S_{1}\right)\right)+a\left(d_{1}-S_{1}\right)$ & $c_{1}-a+h_{2}$ & $c_{2}+h_{2}$ \\
\hline$\Omega_{2}$ & $d_{1}$ & $S_{2}$ & 0 & $c_{1}\left(S_{1}\right)+c_{2}\left(S_{2}\right)+h_{1}\left(S_{1}-d_{1}\right)+p_{2}\left(d_{2}-S_{2}\right)$ & $c_{1}+h_{1}$ & $c_{2}-p_{2}$ \\
\hline$\Omega_{3}$ & $S_{1}$ & $S_{2}$ & 0 & $c_{1}\left(S_{1}\right)+c_{2}\left(S_{2}\right)+p_{1}\left(d_{1}-S_{1}\right)+p_{2}\left(d_{2}-S_{2}\right)$ & $c_{1}-p_{1}$ & $c_{2}-p_{2}$ \\
\hline$\Omega_{4}$ & $S_{1}$ & $d_{2}$ & $S_{2}-d_{2}$ & $c_{1}\left(S_{1}\right)+c_{2}\left(S_{2}\right)+p_{1}\left(d_{1}-S_{1}-\left(S_{2}-d_{2}\right)\right)+a\left(S_{2}-d_{2}\right)$ & $c_{1}-p_{1}$ & $c_{2}-p_{1}+a$ \\
\hline
\end{tabular}

Table B.1: Calculation of the shadow prices for each domain 


\section{Appendix C}

This appendix shows that, in a multiperiod setting with positive flexibility cost, the borderline case with $S_{1}=0$ can never turn out to be optimal when $p_{1}$ lies within the interval $\left[p_{2}, \frac{h_{2} P\left(\Omega_{1}^{M F *}\right)}{P\left(\Omega_{4}^{M F *}\right)}\right]$

Rewriting expression (14) with $p_{1}=\alpha p_{2}(\alpha \geq 0)$ yields:

$$
\overline{c_{1}}=c_{2}+a+(\alpha-1) \frac{h_{2} P\left(\Omega_{1}^{M F *}\right)}{P\left(\Omega_{1}^{M F *}\right)+\alpha P\left(\Omega_{4}^{M F *}\right)}+\alpha(1-\alpha) \frac{p_{2} P\left(\Omega_{4}^{M F *}\right)}{P\left(\Omega_{1}^{M F *}\right)+\alpha P\left(\Omega_{4}^{M F *}\right)}
$$

The borderline case will never be optimal when $\overline{c_{1}}>c_{2}+a$ (as this violates our assumption 1, which requires a positive flexibility cost). Note that $\overline{c_{1}}>c_{2}+a$ is equivalent to $f(\alpha)>0$, with $f(\alpha)=$ $(\alpha-1) h_{2} P\left(\Omega_{1}^{M F *}\right)+\alpha(1-\alpha) p_{2} P\left(\Omega_{4}^{M F *}\right)$.

$f(\alpha)$ has two roots: $\alpha_{1}=1$ and $\alpha_{2}=h_{2} P\left(\Omega_{1}^{M F *}\right) / p_{2} P\left(\Omega_{4}^{M F *}\right)$. Note that $\alpha_{1}$ can be larger or smaller then $\alpha_{2}$; in the remainder, we will assume (without loss of generality) that $\alpha_{1} \leq \alpha_{2}$.

As we know that $f\left(\alpha_{1}\right)=f\left(\alpha_{2}\right)=0$ and $f(\alpha)$ has no other roots, it suffices to determine the sign of the function value in three arbitrary points, chosen in three different intervals (see Table C.1). We opted for the points $\alpha=0, \alpha=\frac{\alpha_{1}+\alpha_{2}}{2}$ and $\alpha=\alpha_{1}+\alpha_{2}$. Table C.1 shows the corresponding $f(\alpha)$.

\begin{tabular}{|c|c|c|}
\hline Interval & $\boldsymbol{\alpha}$ & $\boldsymbol{f}(\boldsymbol{\alpha})$ \\
\hline$\alpha \leq \alpha_{1}$ & 0 & $-h_{2} P\left(\Omega_{1}^{M F *}\right)$ \\
\hline$\alpha_{1} \leq \alpha \leq \alpha_{2}$ & $\frac{\left(1+h_{2} P\left(\Omega_{1}^{M F *}\right) / p_{2} P\left(\Omega_{4}^{M F *}\right)\right)}{2}$ & $\frac{\left(h_{2} P\left(\Omega_{1}^{M F *}\right)-p_{2} P\left(\Omega_{4}^{M F *}\right)\right)^{2}}{4 p_{2} P\left(\Omega_{4}^{M F *}\right)}$ \\
\hline$\alpha \geq \alpha_{2}$ & $1+\frac{h_{2} P\left(\Omega_{1}^{M F *}\right)}{p_{2} P\left(\Omega_{4}^{M F *}\right)}$ & $-h_{2} P\left(\Omega_{1}^{M F *}\right)$ \\
\hline
\end{tabular}

Table C.1: Chosen $\alpha$ values with corresponding $f(\alpha)$ for the three different intervals 
Clearly, $f(\alpha)>0$ only when $\alpha_{1} \leq \alpha \leq \alpha_{2}$. Consequently, we can conclude that the borderline case can never be optimal when $p_{1}$ lies within the interval $\left[p_{2}, \frac{h_{2} P\left(\Omega_{1}^{M F *}\right)}{P\left(\Omega_{4}^{M F *}\right)}\right]$. For $p_{1}$ values outside this interval, the borderline case may turn out to be optimal. 